\title{
A simpler proof for the dimension of the graph of the classical Weierstrass function ${ }^{1}$
}

\author{
Gerhard Keller \\ Department Mathematik, Universität Erlangen-Nürnberg, 91058 Erlangen, Germany.E-mail: keller@math.fau.de
}

Received 16 April 2015; revised 16 July 2015; accepted 18 August 2015

\begin{abstract}
Let $W_{\lambda, b}(x)=\sum_{n=0}^{\infty} \lambda^{n} g\left(b^{n} x\right)$ where $b \geq 2$ is an integer and $g(u)=\cos (2 \pi u)$ (classical Weierstrass function). Building on work by Ledrappier (In Symbolic Dynamics and Its Applications (1992) 285-293), Barański, Bárány and Romanowska (Adv. Math. 265 (2014) 32-59) and Tsujii (Nonlinearity 14 (2001) 1011-1027), we provide an elementary proof that the Hausdorff dimension of $W_{\lambda, b}$ equals $2+\frac{\log \lambda}{\log b}$ for all $\lambda \in\left(\lambda_{b}, 1\right)$ with a suitable $\lambda_{b}<1$. This reproduces results by Barański, Bárány and Romanowska (Adv. Math. 265 (2014) 32-59) without using the dimension theory for hyperbolic measures of Ledrappier and Young (Ann. of Math. (2) 122 (1985) 540-574; Comm. Math. Phys. 117 (1988) 529-548), which is replaced by a simple telescoping argument together with a recursive multi-scale estimate.
\end{abstract}

Résumé. Soit $W_{\lambda, b}(x)=\sum_{n=0}^{\infty} \lambda^{n} g\left(b^{n} x\right)$, où $b \geq 2$ est un nombre entier et $g(u)=\cos (2 \pi u)$ (fonction de Weierstrass classique). En utilisant des idées et résultats de Ledrappier (In Symbolic Dynamics and Its Applications (1992) 285-293), de Barański, Bárány et Romanowska (Adv. Math. 265 (2014) 32-59) et de Tsujii (Nonlinearity 14 (2001) 1011-1027), nous présentons une démonstration élémentaire du fait que la dimension de Hausdorff de $W_{\lambda, b}$ est égale à $2+\frac{\log \lambda}{\log b}$ pour tout $\lambda \in\left(\lambda_{b}, 1\right)$ avec $\lambda_{b}<1$ approprié. Cela reproduit des résultats de Barański, Bárány et Romanowska (Adv. Math. 265 (2014) 32-59) sans utiliser la théorie de dimension des mesures hyperboliques de Ledrappier et Young (Ann. of Math. (2) 122 (1985) 540-574; Comm. Math. Phys. 117 (1988) 529-548), laquelle est remplacée par un argument téléscopique élémentaire conjointement avec une estimation récursive multi-échelle.

MSC: 37D20; 37D45; 37G35; 37H20

Keywords: Weierstrass function; Hausdorff dimension

\section{Introduction}

The classical Weierstrass function $W_{\lambda, b}: \mathbb{I}:=[0,1) \rightarrow \mathbb{R}$ with parameters $b \in \mathbb{N}, \lambda \in(0,1)$ and $b \lambda>1$ is defined by

$$
W_{\lambda, b}(x)=\sum_{n=0}^{\infty} \lambda^{n} \cos \left(2 \pi b^{n} x\right) .
$$

The box dimension of its graph is equal to

$$
D=2+\frac{\log \lambda}{\log b}
$$

\footnotetext{
${ }^{1}$ This work is funded by DFG Grant Ke 514/8-1. I am indebted to Tobias Oertel-Jäger who not only awakened my interest in this problem but made it also a topic of the DFG Scientific Network "Skew Product Dynamics and Multifractal Analysis," and I want to thank Atsuya Otani for pointing out some mistakes in a previous version of this note.
} 
as proved by Kaplan, Mallet-Paret and Yorke in [5]. In 1977, Mandelbrot conjectured in his monograph [10] that $D$ is also the Hausdorff dimension of this graph. Despite many efforts, this conjecture is not yet proved in full generality. Among others it is known to be true for sufficiently large integers [2,3]. The history of the problem and the present state of knowledge are summarized in the introduction to a recent paper by Barański, Bárány and Romanowska [1], in which the authors make a huge step forward by proving that for each integer $b \geq 2$ there exist $\tilde{\lambda}_{b}<\lambda_{b}<1$ such that $D=2+\frac{\log \lambda}{\log b}$ equals the Hausdorff dimension of the graph of $W_{\lambda, b}$ for every $\lambda \in\left(\lambda_{b}, 1\right)$ and for Lebesgue-a.e. $\lambda \in\left(\tilde{\lambda}_{b}, 1\right)$. They determine $\lambda_{b}$ and $\tilde{\lambda}_{b}$ as unique zeroes of certain functions and provide a number of numerical and asymptotic values for them, among others

$$
\lambda_{2}=0.9531, \quad \lambda_{3}=0.7269, \quad \lambda_{4}=0.6083, \quad \text { and } \quad \lim _{b \rightarrow \infty} \lambda_{b}=1 / \pi=0.3183 .
$$

Following Ledrappier [7], they interpret the graph of $W_{\lambda, b}$ as the unique invariant repellor of the dynamical system

$$
\Phi_{\lambda, b}: \mathbb{I} \times \mathbb{R} \rightarrow \mathbb{I} \times \mathbb{R}, \quad \Phi_{\lambda, b}(u, v)=\left(b u \bmod 1, \frac{v-g(u)}{\lambda}\right)
$$

with $g(u)=\cos (2 \pi u)$, and observe that it suffices to show that $D$ is the Hausdorff dimension of the lift of the Lebesgue measure on $\mathbb{I}$ to the graph of $W_{\lambda, b}$, denoted by $\mu_{\lambda, b}$. Then they extend the transformation $u \mapsto b u \bmod 1$ of the first coordinate to an invertible ' $b$-baker' map. ${ }^{2}$ The resulting 3-dimensional system is hyperbolic, and the extension of $\mu_{\lambda, b}$ is a hyperbolic invariant measure for it. This sets the stage to combine dimension results for hyperbolic measures by Ledrappier and Young [8] and [9], an observation by Ledrappier [7] and a transversality estimate by Tsujii [16] to determine $\lambda_{b}$. Finally, additional effort is needed to determine $\tilde{\lambda}_{b}$ based on the transversality approach of Peres and Solomyak [13].

In this note I propose a much more elementary approach to reduce the calculation of the dimension of the graph of the Weierstrass function to the basic estimates provided by Tsujii [16] combined with the numerical estimates by Barański, Bárány and Romanowska [1]. The proof also highlights conceptual similarities to the result by Hunt [4], who studied randomized versions of the Weierstrass graph and proved the validity of the dimension formula for almost all random realizations, see Remark 3.6. An additional benefit of the present approach is that it avoids reference to an argument from [7], that sketches only very briefly how to extend a certain lemma for the piecewise linear function $g(u)=\operatorname{dist}(u, \mathbb{Z})$ to nonlinear functions like $g(u)=\cos (2 \pi u)$ by using techniques from [9]. In passing we also consider the case of the piecewise linear function $g(u)=\operatorname{dist}(u, \mathbb{Z})$ : we provide a short and elementary argument how to reduce the calculation of the dimension of $W_{\lambda, b}$ in this case to the problem of whether an infinite Bernoulli convolution is absolutely continuous, a result that is originally proved in [7]. See Section 3.3.1 for details.

Although this note bypasses completely the general dimension theory of Ledrappier and Young, most proofs in this note are the result of my efforts to understand the basic lines of arguments in the papers mentioned above. The only, but noticeable, exception is Proposition 3.3, that provides a new point of view on how to deal in a more direct way than in [7] with the passage from piecewise linear to nonlinear functions $g(u)$.

Note added in proof: W. Shen [14] refined the arguments from [1] and [15] considerably and proved the dimension formula for all integers $b \geq 2$ and all $\lambda \in\left(b^{-1}, 1\right)$. He mentions that also in his proof Ledrappier's theorem can be replaced by our Proposition 3.3.

\section{The main results}

Throughout this note we use the notation

$$
\gamma:=\frac{1}{b \lambda}<1
$$

Our main results are the new proofs for the following two theorems - not the theorems themselves. The first one is due to Ledrappier [7]:

\footnotetext{
${ }^{2}$ More precisely, they consider the extension of $u \mapsto b u \bmod 1$ by a full one-sided $b$-shift.
} 
Theorem 2.1. Let $g(u)=\operatorname{dist}(u, \mathbb{Z}), b=2$, and let $\lambda \in(0,1)$ be such that the infinite Bernoulli convolution with parameter $\gamma$ has a square-integrable density w.r.t. Lebesgue measure. Then the graph of $W_{\lambda, 2}$ has Hausdorff dimension $D=2+\frac{\log \lambda}{\log 2}$.

Remark 2.2. The infinite Bernoulli convolution with parameter $\gamma$ is the distribution of the random variable $\Theta=$ $\sum_{n=1}^{\infty} \gamma^{n} Z_{n}$, where the $Z_{n}$ are independent random variables with $P\left(Z_{n}=1\right)=P\left(Z_{n}=-1\right)=\frac{1}{2}$. The investigation of $\Theta$ has a long history, see e.g. [11,12] and, for more recent results, also [15].

In particular, the set of parameters $\lambda \in\left(\frac{1}{2}, 1\right)$ for which the corresponding Bernoulli convolution with parameter $\gamma$ has a square integrable density, has full Lebesgue measure in this interval. (It corresponds to $\gamma \in\left(\frac{1}{2}, 1\right)$.) At the expense of only little additional effort our proof extends to the slightly more general case where the distribution of $\Theta$ is only assumed to have dimension 1 , and also integers $b>2$ can be treated in just the same way.

The second theorem is due to Barański, Bárány and Romanowska [1], building upon work of Ledrappier [7] and Tsujii [16]:

Theorem 2.3. Let $g(u)=\cos (2 \pi u)$. For each integer $b \geq 2$ there exists $\lambda_{b}<1$ such that the graph of $W_{\lambda, b}$ described by (1.1) has Hausdorff dimension $D=2+\frac{\log \lambda}{\log b}$ for every $\lambda \in\left(\lambda_{b}, 1\right)$.

Remark 2.4. $\lambda_{b}$ is the unique zero of the function

$$
h_{b}(\lambda)= \begin{cases}\frac{1}{4 \lambda^{2}(2 \lambda-1)^{2}}+\frac{1}{16 \lambda^{2}(4 \lambda-1)^{2}}-\frac{5}{64 \lambda^{2}}+\frac{\sqrt{2}}{2 \lambda}-1 & \text { for } b=2, \\ \frac{1}{(b \lambda-1)^{2}}+\frac{1}{\left(b^{2} \lambda-1\right)^{2}}-\sin ^{2}\left(\frac{\pi}{b}\right) & \text { for } b \geq 3\end{cases}
$$

on the interval $\left(\frac{1}{b}, 1\right)$, see $[1$, Theorem $\mathrm{B}]$.

\section{Proofs}

In Sections 3.1 and 3.2 we recall some observations from [7] and [1], and in Section 3.3 we provide a fresh look at the strong stable manifolds from those references. Section 3.4 contains the telescoping argument already used in a similar situation in [6], and the proof is finished in Sections 3.5 and 3.6 by combining some of the more elementary arguments from [7,16] and [1].

\subsection{The Weierstrass graph as an attractor}

Recall from (1.1) that

$$
\Phi_{\lambda, b}(u, v)=\left(b u \bmod 1, \frac{v-g(u)}{\lambda}\right) .
$$

We are mostly interested in the classical case $g(u)=\cos (2 \pi u)$ and in $g(u)=\operatorname{dist}(u, \mathbb{Z})$ where $g^{\prime}(\xi)=(-1)^{\lfloor 2 \xi\rfloor}$. For notational convenience we denote the map $u \mapsto b u \bmod 1$ by $\tau$ so that $\Phi_{\lambda, b}(u, v)=\left(\tau(u), \frac{v-g(u)}{\lambda}\right)$. Then the Weierstrass function $W=W_{\lambda, b}$ satisfies

$$
\Phi(u, W(u))=(\tau(u), W(\tau(u))) .
$$

In particular,

$$
\lambda W(\tau(u))=W(u)-g(u) .
$$

Denote by $(\xi, x) \mapsto B(\xi, x)$ the $b$-baker map on $\mathbb{I}^{2}$ for the integer $b \geq 2$, i.e.

$$
B(\xi, x)=\left(\tau(\xi), \frac{x+k(\xi)}{b}\right) \quad \text { with } k(\xi)=j \in\{0, \ldots, b-1\} \text { if } \xi \in[j / b,(j+1) / b),
$$


and define $F: \mathbb{I}^{2} \times \mathbb{R} \rightarrow \mathbb{I}^{2} \times \mathbb{R}$ as

$$
F(\xi, x, y)=(B(\xi, x), \lambda y+f(\xi, x)) \quad \text { with } f(\xi, x):=g\left(\frac{x+k(\xi)}{b}\right) .
$$

Then the graph of the Weierstrass function $W=W_{\lambda, b}$, interpreted as a function of $\xi$ and $x$ although it depends on $x$ only, is an invariant attractor for $F$ in the following sense:

$$
\begin{aligned}
F(\xi, x, W(\xi, x)) & =\left(B(\xi, x), \lambda W(x)+g\left(\frac{x+k(\xi)}{b}\right)\right)=\left(B(\xi, x), W\left(\frac{x+k(\xi)}{b}\right)\right) \\
& =(B(\xi, x), W(B(\xi, x))),
\end{aligned}
$$

where the second identity follow from (3.1) with $u=\frac{x+k(\xi)}{b}$. As $F$ has skew-product structure over the base $B$ and as $\left|\frac{\partial F_{3}}{\partial y}\right|=\lambda<1$, the graph of $W$ is an attractor for $F$.

\subsubsection{Notation for orbits}

Given a point $(\xi, x) \in \mathbb{I}^{2}$ we denote by $\left(\xi_{n}, x_{n}\right)$ the point $B^{n}(\xi, x)(n \in \mathbb{Z})$. Note that $\xi_{n}=\tau^{n}(\xi)(n \geq 0)$ and $x_{n}=$ $\tau^{-n}(x)(n \leq 0)$ and that

$$
k\left(\xi_{i}\right)=k\left(x_{i+1}\right) \quad \text { for all } i \in \mathbb{Z} .
$$

We also write

$$
k_{n}(\xi)=\sum_{i=0}^{n-1} b^{i} k\left(\tau^{i} \xi\right) .
$$

For later use we note that

$$
\begin{aligned}
k_{n}(\xi) & =\sum_{i=0}^{n-1} b^{i} k\left(\xi_{i}\right)=b^{n} \sum_{i=0}^{n-1} b^{i-n} k\left(x_{i+1}\right)=b^{n} \sum_{j=0}^{n-1} b^{-j-1} k\left(x_{n-j}\right)=b^{n} \sum_{j=0}^{n-1} b^{-j-1} k\left(\tau^{j} x_{n}\right) \\
& =b^{n} x_{n}-b^{n} \sum_{j=n}^{\infty} b^{-j-1} k\left(x_{n-j}\right)=b^{n} x_{n}-\sum_{i=0}^{\infty} b^{-i-1} k\left(x_{-i}\right)=b^{n} x_{n}-\sum_{i=0}^{\infty} b^{-i-1} k\left(\tau^{i} x\right) \\
& =b^{n} x_{n}-x .
\end{aligned}
$$

In particular,

$$
\begin{aligned}
& k_{n}\left(\xi_{-i}\right)=b^{n} \tau^{i-n}(x)-\tau^{i}(x) \text { for } n \leq i, \\
& k_{n}\left(\xi_{-i}\right)=b^{n} x_{n-i}-\tau^{i}(x) \text { for } n \geq i,
\end{aligned}
$$

and

$$
\frac{x_{n}+k\left(\xi_{n}\right)}{b}=\frac{x+k_{n}(\xi)+b^{n} k\left(\xi_{n}\right)}{b^{n+1}}=\frac{x+k_{n+1}(\xi)}{b^{n+1}}=x_{n+1} .
$$

For comparison with the notation of [1] note also that

$$
x_{n}=\frac{x+k_{n}(\xi)}{b^{n}}=\frac{x}{b^{n}}+\frac{k\left(x_{1}\right)}{b^{n}}+\cdots+\frac{k\left(x_{n}\right)}{b}=\frac{x}{b^{n}}+\frac{k\left(\xi_{0}\right)}{b^{n}}+\cdots+\frac{k\left(\xi_{n-1}\right)}{b} .
$$




\subsection{Stable and unstable manifolds}

Following [1] and also the earlier paper [7], we describe the stable and unstable manifolds of $F$. The derivative DF is well defined except when $\xi \in S:=\{j / b: j=0, \ldots, b-1\}$, namely

$$
\operatorname{DF}(\xi, x, y)=\left(\begin{array}{ccc}
b & 0 & 0 \\
0 & \frac{1}{b} & 0 \\
0 & \frac{\partial f}{\partial x}(\xi, x) & \lambda
\end{array}\right)
$$

The Lyapunov exponents of the corresponding cocycle $\operatorname{are} \log b,-\log b$ and $\log \lambda$. Indeed, they correspond to the invariant vector fields

$$
\left(\begin{array}{l}
1 \\
0 \\
0
\end{array}\right), \quad X(\xi, x, y)=\left(\begin{array}{c}
0 \\
1 \\
-\lambda^{-1} \sum_{n=0}^{\infty} \gamma^{n} \frac{\partial f}{\partial x}\left(B^{n}(\xi, x)\right)
\end{array}\right), \quad \text { and } \quad\left(\begin{array}{l}
0 \\
0 \\
1
\end{array}\right),
$$

where $\gamma=(b \lambda)^{-1}$. Observe that none of these fields depends on the variable $y$, so we write $X(\xi, x)$ henceforth.

Remark 3.1. As $\frac{\partial f}{\partial x}\left(B^{n}(\xi, x)\right)=b^{-1} g^{\prime}\left(\frac{x_{n}+k\left(\xi_{n}\right)}{b}\right)=b^{-1} g^{\prime}\left(x_{n+1}\right)$, the third component of $X$ can be written as

$$
X_{3}(\xi, x)=-\sum_{n=1}^{\infty} \gamma^{n} g^{\prime}\left(x_{n}\right)=-\sum_{n=1}^{\infty} \gamma^{n} g^{\prime}\left(\frac{x+k_{n}(\xi)}{b^{n}}\right)
$$

which is precisely the second component of the field $\mathcal{J}_{x, \mathbf{i}}$ of [1], also denoted $Y_{x, \gamma}(\mathbf{i})$ in that paper.

For each fixed $\xi$, the field $X$ defines the strong stable foliation in the $(x, y)$-plane $H_{\xi}$ over $\xi$. The fibres are parallel graphs over $x$ with uniformly bounded slopes. That means, for all $(\xi, x, y) \in(\mathbb{I} \backslash S) \times \mathbb{I} \times \mathbb{R}$ the fibre through $(\xi, x, y)$ is the graph of a function $\ell_{(\xi, x, y)}^{s s}: \mathbb{I} \rightarrow \mathbb{R}$ that solves the initial value problem

$$
\frac{\partial}{\partial v} \ell_{(\xi, x, y)}^{s s}(v)=X_{3}(\xi, v) \quad \text { and } \quad \ell_{(\xi, x, y)}^{s s}(x)=y .
$$

Denote by $\mathcal{G} \ell_{(\xi, x, y)}^{s s}$ the graph of the function $\ell_{(\xi, x, y)}^{s s}$ in the hyperplane $H_{\xi}$, i.e.

$$
\mathcal{G} \ell_{(\xi, x, y)}^{s s}=\left\{\left(\xi, u, \ell_{(\xi, x, y)}^{s s}(u)\right): u \in \mathbb{I}\right\} .
$$

As the foliation into strong stable fibres is invariant, we have

$$
F\left(\mathcal{G} \ell_{(\xi, x, y)}^{s s}\right) \subseteq \mathcal{G} \ell_{F(\xi, x, y)}^{s s}
$$

\subsection{Distances between strong stable fibres}

Given two points $(\xi, x),\left(\xi, x^{\prime}\right) \in \mathbb{I}^{2}$ we denote by $\left|\Delta_{\xi}\left(x, x^{\prime}\right)\right|$ the vertical distance of the strong stable fibres through the points $(\xi, x, W(x))$ and $\left(\xi, x^{\prime}, W\left(x^{\prime}\right)\right)$, respectively. More precisely,

$$
\begin{aligned}
\Delta_{\xi}\left(x, x^{\prime}\right) & =\ell_{\left(\xi, x^{\prime}, W\left(x^{\prime}\right)\right)}^{s s}-\ell_{(\xi, x, W(x))}^{s s}=\ell_{\left(\xi, x^{\prime}, W\left(x^{\prime}\right)\right)}^{s s}\left(x^{\prime}\right)-\ell_{(\xi, x, W(x))}^{s s}\left(x^{\prime}\right) \\
& =W\left(x^{\prime}\right)-W(x)-\left(\ell_{(\xi, x, W(x))}^{s s}\left(x^{\prime}\right)-\ell_{(\xi, x, W(x))}^{s s}(x)\right) \\
& =W\left(x^{\prime}\right)-W(x)-\int_{x}^{x^{\prime}} X_{3}(\xi, t) d t \\
& =W\left(x^{\prime}\right)-W(x)+\sum_{n=1}^{\infty} \gamma^{n} \int_{x}^{x^{\prime}} g^{\prime}\left(\frac{t+k_{n}(\xi)}{b^{n}}\right) d t .
\end{aligned}
$$




\subsubsection{The piecewise linear case}

In the piecewise linear case $g^{\prime}(u)=(-1)^{\lfloor 2 u\rfloor}$ and $b=2$ we have

$$
\begin{aligned}
g^{\prime}\left(\frac{t+k_{n}(\xi)}{b^{n}}\right) & =g^{\prime}\left(\frac{t}{b^{n}}+\frac{k\left(\xi_{0}\right)}{b^{n}}+\cdots+\frac{k\left(\xi_{n-1}\right)}{b}\right)=g^{\prime}\left(\frac{k\left(\xi_{n-1}\right)}{2}\right) \\
& =(-1)^{k\left(\xi_{n-1}\right)}
\end{aligned}
$$

so that

$$
\begin{aligned}
\Delta_{\xi}\left(x, x^{\prime}\right) & =W\left(x^{\prime}\right)-W(x)+\left(x^{\prime}-x\right) \cdot \sum_{n=1}^{\infty} \gamma^{n}(-1)^{k\left(\xi_{n-1}\right)} \\
& =W\left(x^{\prime}\right)-W(x)+\left(x^{\prime}-x\right) \cdot \Theta(\xi)
\end{aligned}
$$

where $\Theta(\xi):=\sum_{n=1}^{\infty} \gamma^{n}(-1)^{k\left(\xi_{n-1}\right)}$ is an infinite Bernoulli convolution. It is known $[11,15]$ that for Lebesgue-a.e. $\gamma \in\left(\frac{1}{2}, 1\right)$ the distribution of the random variable $\Theta$ has a square-integrable density $h$ w.r.t. Lebesgue measure. For such $\Theta$ the following holds:

Denote Lebesgue measure on $\mathbb{I}^{2}$ by $m^{2}$. For each $\delta>0$ there is $C>0$ such that for each $r>0$ there is a measurable set $E_{r} \subset \mathbb{I}^{2}$ with $m^{2}\left(E_{r}\right) \leq C r^{\delta}$ and the following property: For each $z \in[-1,1] \backslash\{0\}$ and for each measurable family $\left(\mathbb{L}_{x}\right)_{x \in \mathbb{I}}$ of intervals of length $\frac{2 r}{|z|}$,

$$
m^{2}\left\{(\xi, x) \in \mathbb{I}^{2} \backslash E_{r}: \Theta(\xi) \in \mathbb{L}_{x}\right\} \leq r^{1-2 \delta}|z|^{-1} .
$$

Indeed, let $E_{r}=\left\{(\xi, x) \in \mathbb{I}^{2}: h(\Theta(\xi))>r^{-\delta}\right\}$. Then

$$
\begin{aligned}
m^{2}\left(E_{r}\right) & =\int_{\mathbb{I}} \int_{\mathbb{I}} 1_{\left\{(\xi, x): h(\Theta(\xi, x))>r^{-\delta}\right\}} d \xi d x=\int_{\mathbb{R}} 1_{\left\{\theta: h(\theta)>r^{-\delta}\right\}} d\left(m^{2} \circ \Theta^{-1}\right)(\theta) \\
& =\int_{\mathbb{R}} 1_{\left\{\theta: h(\theta)>r^{-\delta}\right\}} h(\theta) d \theta \leq r^{\delta}\|h\|_{2}^{2}
\end{aligned}
$$

and

$$
m^{2}\left\{(\xi, x) \in \mathbb{I}^{2} \backslash E_{r}: \Theta(\xi) \in \mathbb{L}_{x}\right\}=\int_{\left\{\theta: h(\theta) \leq r^{-\delta}\right\}} 1_{\mathbb{L}_{x}}(\theta) h(\theta) d \theta \leq 2 r^{1-\delta}|z|^{-1} .
$$

This is a simplified version of the more subtle Proposition 3.3 that we state and prove below for the case of nonlinear $g$. Readers who want first to see how the proof for the present case is finished, can jump immediately to Section 3.4 and only read that one and Section 3.5.

\subsubsection{The case $g(u)=\cos (2 \pi u)$}

If $g(u)=\cos (2 \pi u)$, then

$$
\begin{aligned}
\int_{x}^{x^{\prime}} g^{\prime}\left(\frac{t+k_{n}(\xi)}{b^{n}}\right) d t & =b^{n}\left(\cos \left(2 \pi \frac{x^{\prime}+k_{n}(\xi)}{b^{n}}\right)-\cos \left(2 \pi \frac{x+k_{n}(\xi)}{b^{n}}\right)\right) \\
& =-2 b^{n} \sin \left(2 \pi \frac{x^{\prime}-x}{2 b^{n}}\right) \sin \left(2 \pi\left(\frac{x^{\prime}+x}{2}+k_{n}(\xi)\right) / b^{n}\right),
\end{aligned}
$$

so that, with $s(t):=(t / 2)^{-1} \sin (2 \pi t / 2)$,

$$
\Delta_{\xi}\left(x, x^{\prime}\right)=W\left(x^{\prime}\right)-W(x)-\left(x^{\prime}-x\right) \sum_{n=1}^{\infty} \gamma^{n} s\left(\frac{x^{\prime}-x}{b^{n}}\right) \sin \left(2 \pi\left(x+\frac{x^{\prime}-x}{2}+k_{n}(\xi)\right) / b^{n}\right) .
$$


With

$$
\Theta_{z}(\xi, x):=\sum_{n=1}^{\infty} \gamma^{n} s\left(\frac{z}{b^{n}}\right) \sin \left(2 \pi\left(x_{n}+\frac{z}{2 b^{n}}\right)\right)
$$

this can be written as

$$
\Delta_{\xi}\left(x, x^{\prime}\right)=W\left(x^{\prime}\right)-W(x)-\left(x^{\prime}-x\right) \cdot \Theta_{x^{\prime}-x}(\xi, x),
$$

because $\frac{x+k_{n}(\xi)}{b^{n}}=x_{n}$, see (3.4).

The function $\Theta_{0}(\xi, x)=-2 \pi \sum_{n=1}^{\infty} \gamma^{n} \sin \left(2 \pi x_{n}\right)$ is, up to some constant factor and different notation, just the function $S(x, \mathbf{i})$ of Barański, Bárány and Romanowska [1]. Denote by $v_{x}:=\left(m \times \delta_{x}\right) \circ \Theta_{0}^{-1}$ the conditional distribution of $\Theta_{0}(\xi, x)$ given $x \in \mathbb{I}$. In [1, Proposition 4.2] the authors verify by some explicit real analysis estimates that a transversality condition from Tsujii's paper [16] is satisfied, so that results from the more elementary Sections 3 and 4 of [16] guarantee the absolute continuity of the $v_{x}$ w.r.t. Lebesgue measure. This leads to the following proposition.

Proposition 3.2. Let $\lambda \in\left(\lambda_{b}, 1\right)$, i.e. $b \gamma \in\left(1, \lambda_{b}^{-1}\right)$. For $m$-a.e. $x \in \mathbb{I}$, the measure $v_{x}$ is absolutely continuous w.r.t. $m$. Its density $h_{x}$ satisfies $H:=\int_{\mathbb{I}}\left\|h_{x}\right\|_{2}^{2} d x<\infty$.

A major technical problem is that this estimate is needed also for $z \neq 0$. One approach could be to imitate Tsujii's recursion from [16], and indeed, one obtains densities $h_{x}$ with $\sup _{|z| \leq 1} \int_{\mathbb{I}}\left\|h_{x}\right\|_{2}^{2} d x<\infty$. But this approach does not provide any local information on the $h_{x}$ uniformly in $z$ : the set of $(\xi, x)$ where $h_{x}(\xi)$ is exceptionally big, might depend on $z$ in a complicated way. Therefore we follow a different approach here. Naively, one can start with comparing $\Theta_{z}$ to $\Theta_{0}$ : it is easily seen that there is a constant $C>0$ such that $\left\|\Theta_{z}-\Theta_{0}\right\|_{\infty} \leq C|z|$. As in later steps of the proof we have to approximate $\Theta_{z}$ by $\Theta_{0}$ up to an error of order $r$ for small $r>0$, this would cover only $|z|<r .{ }^{3}$ However, if one treats a finite part of the sum defining $\Theta_{z}$ separately from the remaining tail, one sees that a tail starting at $n=n_{0}$ varies with $z$ only of the order $\left(\frac{\gamma}{b}\right)^{n_{0}}|z|$. Using this observation recursively we will prove the following result in Section 3.6.

Proposition 3.3. Let $\lambda \in\left(\lambda_{b}, 1\right)$. For each $\eta>0$ there are $\delta \in(0, \eta)$ and $C>0$ such that for each $r \in(0,1)$ there is a measurable set $E_{r} \subset \mathbb{I}^{2}$ with $m^{2}\left(E_{r}\right) \leq C r^{\delta}$ and the following property: For each $z \in[-1,1] \backslash\{0\}$ and for each measurable family $\left(\mathbb{L}_{x}\right)_{x \in \mathbb{I}}$ of intervals of length $\frac{2 r}{|z|}$,

$$
m^{2}\left\{(\xi, x) \in \mathbb{I}^{2} \backslash E_{r}: \Theta_{z}(\xi, x) \in \mathbb{L}_{x}\right\} \leq C r^{1-2 \eta}|z|^{-(1-\eta)}
$$

A crucial ingredient of the proof is the following observation:

Remark 3.4. Recall from (3.4) that $x_{n}=\frac{x}{b^{n}}+\frac{k\left(\xi_{0}\right)}{b^{n}}+\cdots+\frac{k\left(\xi_{n-1}\right)}{b}$. Hence the conditional distribution, given $\left(x, k\left(\xi_{0}\right), \ldots, k\left(\xi_{N-1}\right)\right)$, of

$$
\begin{aligned}
\Theta_{0}\left(B^{N}(\xi, x)\right) & =\Theta_{0}\left(\xi_{N}, x_{N}\right)=-2 \pi \sum_{n=1}^{\infty} \gamma^{n} \sin \left(2 \pi x_{N+n}\right) \\
& =-2 \pi \sum_{n=1}^{\infty} \gamma^{n} \sin \left(2 \pi\left(\frac{x_{N}}{b^{n}}+\frac{k\left(\xi_{N}\right)}{b^{n}}+\frac{k\left(\xi_{N+1}\right)}{b^{n-1}}+\cdots+\frac{k\left(\xi_{N+n-1}\right)}{b}\right)\right)
\end{aligned}
$$

is $v_{x_{N}}$, the distribution of $\Theta_{0}\left(\cdot, x_{N}\right)$, because the $k\left(\xi_{n}\right)$ are independent and uniformly distributed on $\{0, \ldots, b-1\}$.

\footnotetext{
${ }^{3}$ The same problem occurs also in Ledrappier's sketch of a related proof [7]. He solves it by using formulas relating dimensions and exponents of various conditional and projected measures as in [8].
} 


\subsection{Telescoping - a replacement for the Ledrappier-Young argument}

\subsubsection{Neighbourhoods bounded by strong stable fibres}

We define a kind of $\varepsilon$-neighbourhoods of points $(\xi, x, W(x))$ in $(x, y)$-direction. To that end fix a constant $K>0$ (to be determined later) and, for any $\xi \in \mathbb{I}$ and a $b$-adic $\varepsilon$-neighbourhood $I_{N}(x)$ of $x \in \mathbb{I}$ with $\varepsilon=b^{-N}$, let

$$
V_{N}(\xi, x)=\left\{(v, w) \in \mathbb{I} \times \mathbb{R}: v \in I_{N}(x),\left|w-\ell_{(\xi, x, W(x))}^{s s}(v)\right| \leq K b^{-N}\right\} .
$$

The sets $\{\xi\} \times V_{N}(\xi, x)$ are curved quadrilaterals in $H_{\xi}$, which are bounded in $x$-direction by two vertical lines of distance $b^{-N}$ and in $y$-direction by the strong stable fibres through $\left(\xi, x, W(x) \pm K b^{-N}\right)$ (which are parallel!). Denote by $G:=\{(x, W(x)): x \in \mathbb{I}\}$ the graph of $W$, let

$$
A_{N}(\xi, x)=V_{N}(\xi, x) \cap G
$$

and let $\mu$ be the Lebesgue measure $m$ on $\mathbb{I}$ lifted to $G$. We will evaluate the local dimension (in $H_{\xi}$ ) of $\mu$ at $(x, W(x)) \in$ $G$ along $b$-adic neighbourhoods $V_{N}(\xi, x)$, i.e. we are going to determine the limit

$$
\lim _{N \rightarrow \infty} \frac{\log \mu\left(V_{N}(\xi, x)\right)}{\log \left(b^{-N}\right)} .
$$

Observe that this limit, if it exists, does not depend on $\xi$, as the next remark shows among others.

Remark 3.5. As $X_{3}$ is uniformly bounded by some constant $K_{1}$, all $\ell_{(\xi, x, W(x))}^{s s}$ have $K_{1}$ as a common Lipschitz constant. Fixing the constant $K$ as $K_{1}+1$ and choosing $n_{1} \in \mathbb{N}$ such that $b^{n_{1}}>2 K_{1}+1$, elementary geometric arguments show that

$$
V_{N+n_{1}}(\xi, x) \subseteq\left\{(v, w) \in \mathbb{I} \times \mathbb{R}: v \in I_{N}(x),|w-W(x)| \leq b^{-N}\right\} \subseteq V_{N}(\xi, x) .
$$

This proves not only that the limit in (3.9) does not depend on $\xi$, but also that the $V_{N}(\xi, x)$ can be replaced by rectangles of height $2 \cdot 2^{-N}$ over the base $I_{N}(x)$.

Furthermore, for $m$-a.e. $x$, one can replace the dyadic intervals $I_{N}(x)$ by symmetric intervals $I_{N}^{\prime}(x):=[x-$ $\left.2^{-N}, x+2^{-N}\right]$ and hence $V_{N}(\xi, x)$ by $V_{N}^{\prime}(\xi, x):=I_{N}^{\prime}(x) \times I_{N}^{\prime}(W(x))$. Indeed, it is immediate that $V_{N+n_{1}}(\xi, x) \subseteq$ $V_{N}^{\prime}(\xi, x)$ and, by Borel-Cantelli, for $m$-a.e. $x$ there is $N(x) \in \mathbb{N}$ such that $V_{N+\left[2 \log _{2} N\right]}^{\prime}(\xi, x) \subseteq V_{N}(\xi, x)$ for all $N \geq N(x)$.

\subsubsection{The telescoping step}

$F^{-N}\left(\{\xi\} \times V_{N}(\xi, x)\right)$ is the image of the curved quadrilateral $\{\xi\} \times V_{N}(\xi, x)$ in $H_{\xi_{-N}}$ under a map which maps vertical line segments affinely to vertical line segments, strong stable fibres into strong stable fibres, and which leaves the graph of $W$ invariant.

This leads to the following identities: Fix $(\xi, x) \in \mathbb{I}^{2}$ and denote as before $\left(\xi_{-N}, x_{-N}\right)=B^{-N}(\xi, x)$. Then, for every $v \in I_{N}(x)$, we have $B^{-N}(\xi, v)=\left(\xi_{-N}, \tau^{N} v\right)$. As the graph of $W$ is invariant under $F^{N}$ by (3.3), we have

$$
F^{N}\left(\xi_{-N}, \tau^{N} v, W\left(\tau^{N} v\right)\right)=(\xi, v, W(v)),
$$

and as strong stable fibres are mapped into strong stable fibres by $F^{N}$, we also have

$$
F^{N}\left(\xi_{-N}, \tau^{N} v, \ell_{\left(\xi_{-N}, x_{-N}, W\left(x_{-N}\right)\right)}^{s s}\left(\tau^{N} v\right)\right)=\left(\xi, v, \ell_{(\xi, x, W(x))}^{s s}(v)\right) .
$$

Recall from (3.2) that, for fixed $v$, the map $y \mapsto \pi_{3}\left(F^{N}\left(\xi_{-N}, \tau^{N} v, y\right)\right)$ is affine with slope $\lambda^{N}$. Therefore the two preceding identities imply that

$$
\begin{aligned}
\left|W(v)-\ell_{(\xi, x, W(x))}^{s s}(v)\right| & \left.=\lambda^{N} \cdot \mid W\left(\tau^{N} v\right)-\ell_{(\xi-N}^{s s}, x_{-N}, W\left(x_{-N}\right)\right) \\
& =\lambda^{N} \cdot\left|\ell_{\left(\xi_{-N}, \tau^{N} v, W\left(\tau^{N} v\right)\right)}^{s s}\left(\tau^{N} v\right)-\ell_{\left(\xi_{-N}, x_{-N}, W\left(x_{-N}\right)\right)}^{s s}\left(\tau^{N} v\right)\right| \\
& =\lambda^{N} \cdot\left|\Delta_{\xi_{-N}}\left(x_{-N}, \tau^{N} v\right)\right| .
\end{aligned}
$$


Now the linearity of $\left.\tau^{N}\right|_{I_{N}(x)}$ allows the following telescoping step:

$$
\begin{aligned}
\frac{\mu\left(V_{N}(\xi, x)\right)}{m\left(I_{N}(x)\right)} & =\frac{m\left(\left\{v \in I_{N}(x):\left|W(v)-\ell_{(\xi, x, W(x))}^{s s}(v)\right| \leq K b^{-N}\right\}\right)}{m\left(I_{N}(x)\right)} \\
& =\frac{m\left(\left\{\tau^{N} v: v \in I_{N}(x),\left|\Delta_{\xi_{-N}}\left(x_{-N}, \tau^{N} v\right)\right| \leq K(\lambda b)^{-N}\right\}\right)}{m\left(\tau^{N}\left(I_{N}(x)\right)\right)} \\
& =\frac{m\left(\left\{x^{\prime} \in \mathbb{I}:\left|\Delta_{\xi_{-N}}\left(x_{-N}, x^{\prime}\right)\right| \leq K \gamma^{N}\right\}\right)}{m(\mathbb{I})}
\end{aligned}
$$

so that, observing $m\left(I_{N}(x)\right)=b^{-N}$,

$$
\lim _{N \rightarrow \infty} \frac{\log \mu\left(V_{N}(\xi, x)\right)}{\log \left(b^{-N}\right)}=1+\lim _{N \rightarrow \infty} \frac{\log m\left\{x^{\prime} \in \mathbb{I}:\left|\Delta_{\xi_{-N}}\left(x_{-N}, x^{\prime}\right)\right| \leq K \gamma^{N}\right\}}{\log \left(b^{-N}\right)}
$$

provided the limits exist. This corresponds to identity (2.3) in [1], which states that $\operatorname{dim} \mu=1+\frac{\log \gamma}{\log b^{-1}} \cdot \operatorname{dim} v_{x, \mathbf{i}}$ for certain probabilities $v_{x, \mathbf{i}}$. Indeed, the remaining task in that paper, namely to show that $\operatorname{dim} v_{x, \mathbf{i}} \geq 1$, corresponds in our approach to showing that

$$
\liminf _{N \rightarrow \infty} \frac{\log m\left\{x^{\prime} \in \mathbb{I}:\left|\Delta_{\xi_{-N}}\left(x_{-N}, x^{\prime}\right)\right| \leq K \gamma^{N}\right\}}{\log \left(\gamma^{N}\right)} \geq 1 \quad \text { for } m^{2} \text {-a.e. }(\xi, x) \in \mathbb{I}^{2} .
$$

We prove this in Section 3.5. Indeed, for typical $(\xi, x)$, the distribution of the random variable $\Delta_{\xi_{-N}}\left(x_{-N}, \cdot\right)$ is closely related to the $v_{x, \mathbf{i}}$ of $[1]$.

Remark 3.6. Instead of projecting along strong stable fibres $\ell_{(\xi, x, W(x))}^{s s}$ that depend on the additional variable $\xi$, one could as well choose a new coordinate system for each $\xi$, describe the Weierstrass function $W$ in this new coordinate system (resulting in a transformed version $W_{\xi}$ of $W$ ) and project the lifts of Lebesgue measure to the graphs of the $W_{\xi}$, horizontally to the real axis. These projected measures would typically be different one from each other (they depend on $\xi)$, but the arguments above show that they all have the same dimension. In this sense our approach is equivalent to determining the dimension of the graph of $W_{\xi}$ for almost all realisations of this random collection of graphs. For Weierstrass graphs with random phase shifts this was done by Hunt [4]. The difference to our situation is that Hunt introduced additional external randomness to the problem so that, for exceptional realizations, his random graphs may have a dimension different from the typical one. In contrast to that, in our case the randomness is generated by the dynamics itself, namely by the unstable coordinate of the underlying baker map, and the construction guarantees that there are no exceptional realizations.

\subsection{A Marstrand projection estimate}

For our further discussion we use the assumption, covering both theorems, that the parameter $\gamma$ is such that the random variables $\Theta_{z}$ on $\left(\mathbb{I}^{2}, m^{2}\right)$ have distributions of dimension 1 in the sense that they obey the conclusion of Proposition 3.3. For the classical Weierstrass function with $\lambda \in\left(\lambda_{b}, 1\right)$ we prove this proposition at the end of this note. In the piecewise linear case where $\Theta_{z}(\xi, x)=\Theta(\xi)$ is an infinite Bernoulli convolution, this is an additional assumption satisfied for Lebesgue-almost $\gamma \in\left(\frac{1}{2}, 1\right)$ as discussed around Equation (3.6).

The following argument is inspired by [7]. Let $\eta>0$ and let $\delta \in(0, \eta), C>0$ and the sets $E_{r} \subseteq \mathbb{I}^{2}$ be as in Proposition 3.3. Let $\mathbb{A}=\left\{(\xi, x, z) \in \mathbb{I}^{2} \times[-1,1]: 0 \leq x+z \leq 1\right\}$ and $J_{r, x, z}:=\left[\frac{W(x+z)-W(x)}{z}-\frac{r}{|z|}, \frac{W(x+z)-W(x)}{z}+\right.$ $\left.\frac{r}{|z|}\right]$. Then

$$
\begin{aligned}
& m^{3}\left\{\left(\xi, x, x^{\prime}\right) \in \mathbb{I}^{3}:(\xi, x) \notin E_{r},\left|\Delta_{\xi}\left(x, x^{\prime}\right)\right| \leq r\right\} \\
& \quad=m^{3}\left\{\left(\xi, x, x^{\prime}\right) \in \mathbb{I}^{3}:(\xi, x) \notin E_{r},\left|\Theta_{x^{\prime}-x}(\xi, x) \cdot\left(x^{\prime}-x\right)-\left(W\left(x^{\prime}\right)-W(x)\right)\right| \leq r\right\} \\
& \quad=m^{3}\left\{(\xi, x, z) \in \mathbb{A}:(\xi, x) \notin E_{r}, \Theta_{z}(\xi, x) \in J_{r, x, z}\right\}
\end{aligned}
$$




$$
\begin{aligned}
& =\int_{-1}^{1} m^{2}\left\{(\xi, x) \in \mathbb{I}^{2} \backslash E_{r}:(\xi, x, z) \in \mathbb{A}, \Theta_{z}(\xi, x) \in J_{r, x, z}\right\} d z \\
& \leq C \int_{-1}^{1} r^{1-2 \eta}|z|^{-(1-\eta)} d z \quad \text { (by Proposition 3.3) } \\
& \leq C r^{1-2 \eta} .
\end{aligned}
$$

Here and in the sequel, $C$ denotes a generic constant whose value may change from occurrence to occurrence and depend on the fixed quatities $\eta, \delta$ and $\ell$. Therefore, writing again $\left(\xi_{-N}, x_{-N}\right)$ for $B^{-N}(\xi, x)$ and using the $B$-invariance of $m^{2}$

$$
\begin{aligned}
& m^{2}\left\{(\xi, x) \in \mathbb{I}^{2}: m\left\{x^{\prime} \in \mathbb{I}:\left|\Delta_{\xi_{-N}}\left(x_{-N}, x^{\prime}\right)\right| \leq r\right\} \geq r^{1-3 \eta}\right\} \\
& \quad=m^{2}\left\{(\xi, x) \in \mathbb{I}^{2}: m\left\{x^{\prime} \in \mathbb{I}:\left|\Delta_{\xi}\left(x, x^{\prime}\right)\right| \leq r\right\} \geq r^{1-3 \eta}\right\} \\
& \leq m^{2}\left(E_{r}\right)+m^{2}\left\{(\xi, x) \in \mathbb{I}^{2} \backslash E_{r}: m\left\{x^{\prime} \in \mathbb{I}:\left|\Delta_{\xi}\left(x, x^{\prime}\right)\right| \leq r\right\} \geq r^{1-3 \eta}\right\} \\
& \quad \leq C r^{\delta}+r^{-(1-3 \eta)} \int_{\mathbb{I}^{2} \backslash E_{r}} m\left\{x^{\prime} \in \mathbb{I}:\left|\Delta_{\xi}\left(x, x^{\prime}\right)\right| \leq r\right\} d m^{2}(\xi, x) \\
& =C r^{\delta}+r^{-(1-3 \eta)} m^{3}\left\{\left(\xi, x, x^{\prime}\right) \in \mathbb{I}^{3}:(\xi, x) \notin E_{r},\left|\Delta_{\xi}\left(x, x^{\prime}\right)\right| \leq r\right\} \\
& \leq C r^{\delta}+C r^{-(1-3 \eta)} r^{1-2 \eta} \\
& \leq C r^{\delta} .
\end{aligned}
$$

By Borel-Cantelli we thus conclude with $r=K \gamma^{N}$ that

$$
\limsup _{N \rightarrow \infty} \gamma^{-(1-3 \eta) N} m\left\{x^{\prime} \in \mathbb{I}:\left|\Delta_{\xi_{-N}}\left(x_{-N}, x^{\prime}\right)\right| \leq K \gamma^{N}\right\} \leq K^{1-3 \eta}
$$

for $m^{2}$-a.e. $(\xi, x) \in \mathbb{I}^{2}$. On a logarithmic scale this implies

$$
\liminf _{N \rightarrow \infty} \frac{\log m\left\{x^{\prime} \in \mathbb{I}^{1}:\left|\Delta_{\xi_{-N}}\left(x_{-N}, x^{\prime}\right)\right| \leq K \gamma^{N}\right\}}{\log \left(b^{-N}\right)} \geq(1-3 \eta) \frac{\log \gamma}{\log b^{-1}},
$$

and as this holds for all $\eta>0$, it proves (3.12) and thus finishes the proofs of Theorems 2.1 and 2.3.

\subsection{Proof of Proposition 3.3}

Let $\eta>0$ and $\alpha:=\frac{\log \gamma}{\log (\gamma / b)}<1$. Choose $\ell \in \mathbb{N}$ such that $\alpha^{\ell}<\eta$ and let $\delta=\frac{\eta}{2 \ell}$.

Given $r \in(0,1)$ and $z \in[-1,1] \backslash\{0\}$, let $r_{z}:=\frac{2 r}{|z|}$. Observe that the claim (3.8) of the proposition is trivial if $|z| \leq 2 r$. So we may assume that $2 r<|z| \leq 1$ and hence that $r_{z} \in[2 r, 1)$. Next let $n_{\ell}:=\left\lceil\frac{\log r_{z}}{\log \gamma}\right\rceil$ and $n_{k}:=\left\lceil\alpha n_{k+1}\right\rceil$ $(k=\ell-1, \ldots, 0)$. Observe that $n_{0} \leq n_{1} \leq \cdots \leq n_{\ell} \leq N:=\left\lceil\frac{\log (2 r)}{\log \gamma}\right\rceil$. On the other hand,

$$
n_{k} \geq \alpha n_{k+1} \geq \cdots \geq \alpha^{\ell-k} n_{\ell} \geq \alpha^{\ell-k} \frac{\log r_{z}}{\log \gamma} \quad(k=0, \ldots, \ell),
$$

so that

$$
\gamma^{n_{k}}=r_{z}^{\left(\log \gamma / \log r_{z}\right) n_{k}} \leq r_{z}^{\alpha^{\ell-k}} \text { and }\left(\frac{\gamma}{b}\right)^{n_{k-1}} \leq\left(\frac{\gamma}{b}\right)^{\alpha n_{k}}=\gamma^{n_{k}} \quad \text { for } k=1, \ldots, \ell .
$$

We will also use the fact that $n_{k} \leq \alpha n_{k+1}+1$, which yields by induction

$$
n_{k} \leq \alpha^{\ell-k} n_{\ell}+\frac{1-\alpha^{\ell-k}}{1-\alpha} \text { for } k=\ell, \ldots, 0 .
$$


For $k=0, \ldots, \ell$ define truncated versions of $\Theta_{z}$,

$$
\Theta_{z, k}(\xi, x):=\sum_{n=1}^{n_{k}} \gamma^{n} s\left(\frac{z}{b^{n}}\right) \sin \left(2 \pi\left(x_{n}+\frac{z}{2 b^{n}}\right)\right),
$$

and also rescaled increments

$$
\begin{aligned}
\Delta_{z, k}(\xi, x) & :=\gamma^{-n_{k-1}}\left(\Theta_{z, k}(\xi, x)-\Theta_{z, k-1}(\xi, x)\right) \\
& =\sum_{n=1}^{d_{k}} \gamma^{n} s\left(\frac{z / b^{n_{k-1}}}{b^{n}}\right) \sin \left(2 \pi\left(x_{n+n_{k-1}}+\frac{z}{2 b^{n+n_{k-1}}}\right)\right),
\end{aligned}
$$

where $d_{k}:=n_{k}-n_{k-1}$. Then

$$
\begin{aligned}
& \left\|\Theta_{z}-\Theta_{z, \ell}\right\|_{\infty} \leq C \gamma^{n_{\ell}}, \\
& \left\|\Theta_{0}\right\|_{\infty} \leq C \text { and }\left\|\Delta_{0, k}-\Theta_{0} \circ B^{n_{k-1}}\right\|_{\infty} \leq C \gamma^{d_{k}},
\end{aligned}
$$

and

$$
\begin{aligned}
\left\|\Theta_{z, k}-\left(\Theta_{z, k-1}+\gamma^{n_{k-1}} \Theta_{0} \circ B^{n_{k-1}}\right)\right\|_{\infty} & =\gamma^{n_{k-1}}\left\|\Delta_{z, k}-\Theta_{0} \circ B^{n_{k-1}}\right\|_{\infty} \\
& \leq \gamma^{n_{k-1}}\left(\left\|\Delta_{z, k}-\Delta_{0, k}\right\|_{\infty}+C \gamma^{d_{k}}\right) \\
& \leq C \gamma^{n_{k-1}}\left(b^{-n_{k-1}}+\gamma^{d_{k}}\right) \leq C \gamma^{n_{k}}
\end{aligned}
$$

by (3.13). From now on we denote by $C$ a fixed constant such that these last estimates are satisfied for $k=1, \ldots, \ell$. Observe that $C$ does neither depend on $r$ nor on $z$.

We have a closer look at measurability properties of $\Delta_{0, k}, \Theta_{0} \circ B^{n_{k-1}}$ and $\Theta_{z, k-1}$ :

$\triangleright \Delta_{0, k}$ depends on $(\xi, x)$ only through $x_{n_{k-1}+1}, \ldots, x_{n_{k}}$, i.e. through $x_{n_{k-1}}, k\left(\xi_{n_{k-1}}\right), \ldots, k\left(\xi_{n_{k}-1}\right)$.

$\triangleright$ The conditional distribution of $\Theta_{0}\left(B^{n_{k-1}}(\xi, x)\right)=-2 \pi \sum_{n=1}^{\infty} \gamma^{n} \sin \left(2 \pi x_{n+n_{k-1}}\right)$ given $x_{n_{k-1}}$ is $v_{x_{n_{k-1}}}(\operatorname{Re}-$ mark 3.4). It has density $h_{x_{n_{k-1}}}$ with respect to Lebesgue measure $m$, because $h_{x}=\frac{d v_{x}}{d m}$ exists for $m$-a.e. $x$ by Proposition 3.2.

$\triangleright \Theta_{z, k-1}$ depends on $(\xi, x)$ only through $x_{1}, \ldots, x_{n_{k-1}}$, i.e. through $x, k\left(\xi_{0}\right), \ldots, k\left(\xi_{n_{k-1}-1}\right)$.

In the rest of the proof we use constants $C_{k}=(2(\ell-k)+1) C, k=0, \ldots, \ell$, where $C>0$ is the constant fixed above. For $j=3,4,5$ let $R_{k, j}=j C+2 C_{k}$ and define probability kernels

$$
\varphi_{k, j}(t)=\frac{1}{2 R_{k, j} \gamma^{d_{k}}} 1_{\left[-R_{k, j} \gamma^{d_{k}, R_{k, j} \gamma^{\left.d_{k}\right]}}(t)\right.}
$$

It is easily checked that for $j=3,4,|\eta| \leq C \gamma^{d_{k}}$ and each nonnegative $h \in L_{m}^{1}(\mathbb{R})$ holds

$$
h * \varphi_{k, j}(u+\eta) \leq 2 h * \varphi_{k, j+1}(u)
$$

Now let

$$
E_{r, k}(z):=\left\{(\xi, x) \in \mathbb{I}^{2}: h_{x_{n_{k-1}}} * \varphi_{k, 4}\left(\Delta_{0, k}(\xi, x)\right)>r^{-2 \delta}\right\},
$$

and $G_{r, k}(z):=\mathbb{I}^{2} \backslash\left(E_{r, 1}(z) \cup \cdots \cup E_{r, k}(z)\right)$. (The argument $z$ reminds of the fact that the sequence $n_{0} \leq n_{1} \leq \cdots \leq$ $n_{\ell} \leq N$ depends on $z$ - but not the common upper bound $N$ !) Observe also that

$\triangleright 1_{E_{r, k}(z)}(\xi, x)$ depends on $(\xi, x)$ only through $x_{n_{k-1}}$ and through $\Delta_{0, k}(\xi, x)$, and hence only through $x, k\left(\xi_{0}\right), \ldots$, $k\left(\xi_{n_{k}-1}\right)$, so that

$\triangleright 1_{G_{r, k-1}(z)}(\xi, x)$ depends on $(\xi, x)$ only through $x, k\left(\xi_{0}\right), \ldots, k\left(\xi_{n_{k-1}-1}\right)$. 
Finally let $E_{r}$ be the union of all such sets $E_{r, k}(z)$ where the union extends over all $k=0, \ldots, \ell$ and all $z \in[2 r, 1)$. A very crude estimate shows that at most $(\ell+1) N^{\ell+1}$ different sets $E_{r, k}(z)$ can contribute to this union. At the end of the proof we will show that there is a constant $C^{\prime}>0$ such that

$$
m^{2}\left(E_{r, k}(z)\right) \leq C^{\prime} r^{2 \delta} \quad \text { for all such sets } E_{r, k}(z) .
$$

Recalling that $N=\left\lceil\frac{\log (2 r)}{\log \gamma}\right\rceil$ and that the choice of $\ell$ did neither depend on $r$ nor on $z$, this shows that $m^{2}\left(E_{r}\right) \leq C^{\prime \prime} r^{\delta}$ as claimed.

Now let $\left(\mathbb{L}_{x}\right)_{x \in \mathbb{I}}$ be a measurable family of intervals of length $r_{z}\left(\approx \gamma^{n_{\ell}}\right)$, and denote by $\mathbb{L}_{x}(t)$ the $t$-neighbourhood of $\mathbb{L}_{x}$. Then, by (3.14),

$$
m^{2}\left\{(\xi, x) \in \mathbb{I}^{2} \backslash E_{r}: \Theta_{z}(\xi, x) \in \mathbb{L}_{x}\right\} \leq m^{2}\left\{(\xi, x) \in G_{r, \ell}(z): \Theta_{z, \ell}(\xi, x) \in \mathbb{L}_{x}\left(C_{\ell} \gamma^{n_{\ell}}\right)\right\} .
$$

Next we prove for all $k=\ell, \ldots, 1$ that

$$
\begin{aligned}
& m^{2}\left\{(\xi, x) \in G_{r, k}(z): \Theta_{z, k}(\xi, x) \in \mathbb{L}_{x}\left(C_{k} \gamma^{n_{k}}\right)\right\} \\
& \quad \leq r^{-2 \delta} 4 R_{k, 3} \gamma^{d_{k}} \cdot m^{2}\left\{(\xi, x) \in G_{r, k-1}(z): \Theta_{z, k-1}(\xi, x) \in \mathbb{L}_{x}\left(C_{k-1} \gamma^{n_{k-1}}\right)\right\} .
\end{aligned}
$$

Indeed, by (3.16) and the definition of the sets $G_{r, k}(z)$,

$$
\begin{aligned}
& m^{2}\left\{(\xi, x) \in G_{r, k}(z): \Theta_{z, k}(\xi, x) \in \mathbb{L}_{x}\left(C_{k} \gamma^{n_{k}}\right)\right\} \\
& \quad \leq m^{2}\left\{(\xi, x) \in G_{r, k-1}(z) \backslash E_{r, k}(z): \Theta_{z, k-1}(\xi, x)+\gamma^{n_{k-1}} \Theta_{0} \circ B^{n_{k-1}}(\xi, x) \in \mathbb{L}_{x}\left(\left(C+C_{k}\right) \gamma^{n_{k}}\right)\right\} .
\end{aligned}
$$

Observing (3.15) and the fact that $2 C+C_{k}=C_{k-1}$, this estimate can be continued by

$$
\begin{aligned}
\leq m^{2}\left\{(\xi, x) \in G_{r, k-1}(z) \backslash E_{r, k}(z): \Theta_{z, k-1}(\xi, x) \in \mathbb{L}_{x}\left(C_{k-1} \gamma^{n_{k-1}}\right)\right. \text { and } \\
\\
\left.\Theta_{0} \circ B^{n_{k-1}}(\xi, x) \in \gamma^{-n_{k-1}}\left(\mathbb{L}_{x}\left(\left(C+C_{k}\right) \gamma^{n_{k}}\right)-\Theta_{z, k-1}(\xi, x)\right)\right\},
\end{aligned}
$$

and as $1_{G_{r, k-1}(z)}(\xi, x)$ and $\Theta_{z, k-1}(\xi, x)$ depend on $(\xi, x)$ only through $x, k\left(\xi_{0}\right), \ldots, k\left(\xi_{n_{k-1}-1}\right)$, this is equal to

$$
\begin{array}{r}
=\int_{G_{r, k-1}(z) \cap\left\{\Theta _ { z , k - 1 } \in \mathbb { L } _ { . } \left(C_{k-1} \gamma^{\left.\left.n_{k-1}\right)\right\}}\right.\right.} m^{2}\left(\left\{\Theta_{0} \circ B^{n_{k-1}}(\xi, x) \in \mathbb{J}_{(\xi, x)}\right\} \backslash E_{r, k}(z)\right. \\
\left.\mid x, k\left(\xi_{0}\right), \ldots, k\left(\xi_{n_{k-1}-1}\right)\right) d m^{2}(\xi, x),
\end{array}
$$

where $\mathbb{J}_{(\xi, x)}=\gamma^{-n_{k-1}}\left(\mathbb{L}_{x}\left(\left(C+C_{k}\right) \gamma^{n_{k}}\right)-\Theta_{z, k-1}(\xi, x)\right)$ is an interval of length at most $R_{k, 3} \gamma^{d_{k}}=\left(3 C+2 C_{k}\right) \gamma^{d_{k}}$, and $\tilde{\mathbb{J}}_{x}:=\mathbb{J}_{B^{-n_{k-1}(\xi, x)}}$ depends on $(\xi, x)$ only through $x$. Observing the definition of the set $E_{r, k}(z)$ in (3.18), an elementary calculation with conditional probabilities and their behaviour under measure preserving maps shows that the conditional probability in the last integral equals

$$
m^{2}\left(\left\{\Theta_{0}(\xi, x) \in \tilde{\mathbb{J}}_{x}\right\} \cap\left\{h_{x} * \varphi_{k, 4}\left(\Delta_{0, k}\left(B^{-n_{k-1}}(\xi, x)\right)\right) \leq r^{-2 \delta}\right\} \mid x\right) \circ B^{n_{k-1}} .
$$

In view of (3.15) and (3.17) this can be estimated by

$$
\begin{aligned}
& \leq m^{2}\left(\left\{\Theta_{0}(\xi, x) \in \tilde{\mathbb{J}}_{x}\right\} \cap\left\{h_{x} * \varphi_{k, 3}\left(\Theta_{0}(\xi, x)\right) \leq 2 r^{-2 \delta}\right\} \mid x\right) \circ B^{n_{k-1}} \\
& =\left.\int_{\tilde{\mathbb{J}}_{x} \cap\left\{h_{x} * \varphi_{k, 3} \leq 2 r^{-2 \delta}\right\}} h_{x}(\theta) d \theta\right|_{x=x_{n_{k-1}}} \\
& \leq \begin{cases}0 & \text { if } \tilde{\mathbb{J}}_{x_{n_{k-1}}} \cap\left\{h_{x_{n_{k-1}}} * \varphi_{k, 3} \leq 2 r^{-2 \delta}\right\}=\varnothing, \\
2 R_{k, 3} \gamma^{d_{k}} \cdot h_{x_{n_{k-1}}} * \varphi_{k, 3}(\tilde{\theta}) & \text { if } \tilde{\theta} \in \tilde{\mathbb{J}}_{x_{n_{k-1}}} \cap\left\{h_{x_{n_{k-1}}} * \varphi_{k, 3} \leq 2 r^{-2 \delta}\right\}\end{cases} \\
& \leq r^{-2 \delta} 4 R_{k, 3} \gamma^{d_{k},}
\end{aligned}
$$


where we used the fact that the conditional distribution of $\Theta_{0}$ given $x$ has density $h_{x}$. This finishes the proof of the recursive estimate (3.21). Combining that estimate with (3.20) we obtain

$$
\begin{aligned}
m^{2}\left\{(\xi, x) \in \mathbb{I}^{2} \backslash E_{r}: \Theta_{z}(\xi, x) \in \mathbb{L}_{x}\right\} & \leq \text { const } r^{-2 \ell \delta} \gamma^{n_{\ell}-n_{0}} \\
& \leq \text { const } r^{-\eta} r_{z}^{1-\eta} \leq \text { const } r^{1-2 \eta}|z|^{-(1-\eta)},
\end{aligned}
$$

where we used that $\eta=2 \ell \delta$ and $n_{\ell}-n_{0} \geq n_{\ell}\left(1-\alpha^{\ell}\right) \geq n_{\ell}(1-\eta)$. As the constant does neither depend on $r$ nor on $z$ (its dependence on the fixed integer $\ell$ does not hurt), this proves the claim (3.8) of Proposition 3.3.

It remains to prove estimate (3.19):

$$
\begin{aligned}
& m^{2}\left\{(\xi, x) \in \mathbb{I}^{2}: h_{x_{n_{k-1}}} * \varphi_{k, 4}\left(\Delta_{0, k}(\xi, x)\right)>r^{-2 \delta}\right\} \\
& \quad=m^{2}\left\{(\xi, x) \in \mathbb{I}^{2}: h_{x} * \varphi_{k, 4}\left(\Delta_{0, k} \circ B^{-n_{k-1}}(\xi, x)\right)>r^{-2 \delta}\right\} \\
& \quad \leq r^{2 \delta} \int_{\mathbb{I}} \int_{\mathbb{I}} h_{x} * \varphi_{k, 4}\left(\Delta_{0, k} \circ B^{-n_{k-1}}(\xi, x)\right) d \xi d x \\
& \leq 2 r^{2 \delta} \int_{\mathbb{I}} \int_{\mathbb{I}} h_{x} * \varphi_{k, 5}\left(\Theta_{0}(\xi, x)\right) d \xi d x \quad(\text { by }(3.15) \text { and }(3.17)) \\
& =2 r^{2 \delta} \int_{\mathbb{I}} \int_{\mathbb{R}} h_{x} * \varphi_{k, 5}(\theta) h_{x}(\theta) d \theta d x \\
& \quad \leq 2 r^{2 \delta} \int_{\mathbb{I}}\left\|h_{x} * \varphi_{k, 5}\right\|_{2}\left\|h_{x}\right\|_{2} d x \leq 2 H r^{2 \delta},
\end{aligned}
$$

where we used the fact that convolution with a probability kernel cannot increase the $L_{m}^{2}$-norm of a function. This finishes the proof of Proposition 3.3.

\section{References}

[1] K. Barański, B. Bárány and J. Romanowska. On the dimension of the graph of the classical Weierstrass function. Adv. Math. 265 (2014) 32-59. MR3255455

[2] L. Biacino. On the Hausdorff dimension of the graph of a Weierstrass type function. Stud. Univ. Babeş-Bolyai Math. 56 (4) (2011) 7-17. MR2896736

[3] S. Fu. On the Hausdorff dimension of the graph of the Weierstrass function. Far East J. Dyn. Syst. 17 (2011) 85-137. MR2952868

[4] B. Hunt. The Hausdorff dimension of graphs of Weierstrass functions. Proc. Amer. Math. Soc. 126 (3) (1998) 791-800. MR1452806

[5] J. Kaplan, J. Mallet-Paret and J. Yorke. The Lyapunov dimension of a nowhere differentiable attracting torus. Ergodic Theory Dynam. Systems 4 (1984) 261-281. MR0766105

[6] G. Keller. Stability index for chaotically driven concave maps. J. Lond. Math. Soc. (2) 89 (2014) 603-622. MR3188635

[7] F. Ledrappier. On the dimension of some graphs. In Symbolic Dynamics and Its Applications (New Haven, CT, 1991) 285-293. Contemp. Math. 135, 1992. MR1185095

[8] F. Ledrappier and L.-S. Young. The metric entropy of diffeomorphisms. II. Relations between entropy, exponents and dimension. Ann. of Math. (2) 122 (1985) 540-574. MR0819557

[9] F. Ledrappier and L.-S. Young. Dimension formula for random transformations. Comm. Math. Phys. 117 (1988) 529-548. MR0953818

[10] B. Mandelbrot. Fractals: Form, Chance, and Dimension. Freeman, San Francisco, 1977. MR0471493

[11] Y. Peres and W. Schlag. Smoothness of projections, Bernoulli convolutions, and the dimension of exceptions. Duke Math. J. 102 (2) (2000) 193-251. MR1749437

[12] Y. Peres, W. Schlag and B. Solomyak. Sixty years of Bernoulli convolutions. In Fractal Geometry and Stochastics II 1-28. Birkhäuser, Basel, 2000. MR1785620

[13] Y. Peres and B. Solomyak. Absolute continuity of Bernoulli convolutions, a simple proof. Math. Res. Lett. 3 (1996) 231-239. MR1386842

[14] W. Shen. Hausdorff dimension of the graphs of the classical Weierstrass functions. Available at arXiv:1505.03986.

[15] P. Shmerkin. On the exceptional set for absolute continuity of Bernoulli convolutions. Geom. Funct. Anal. 24 (3) (2014) 946-958. MR3213835

[16] M. Tsujii. Fat solenoidal attractors. Nonlinearity 14 (2001) 1011-1027. MR1862809 